\title{
Trastornos Neuropsiquiátricos Autoinmunes Pediátricos Asociados a Estreptococo: Presentación de un caso.
}

\author{
Pediatric Autoimmune Neuropsychiatric Disorders Associated With Streptococcal \\ Infections: Presentation of a case
}

\section{Oscar Iván Salinas*, Josué Joaquín Gómez**, Mario Velásquez ${ }^{* * *}$}

\section{RESUMEN}

Los trastornos neuropsiquiátricos autoinmunes pediátricos asociados a estreptococo (PANDAS) fueron creados para describir un subgrupo teórico de casos de trastornos obsesivo compulsivos (TOC) y / o tics en los que el inicio o la exacerbación de los síntomas es desencadenado por infección del estreptococo hemolítico del grupo A (SBHGA) ; Si bien aún no hay datos de incidencia se estima que el trastorno afecta aproximadamente al $1 \%$ de niños menores de 12 años, porque es menos probable que tengan anticuerpos contra el estreptococo y probablemente esté infradiagnosticado. La existencia de este trastorno ha generado considerables avances clínicos y científicos, así como controversia. Fisiopatológicamente, PANDAS se propone ser un trastorno autoinmune postestreptocócico similar a corea de Sydenham, hipotetizandose mas específicamente que los tics y la sintomatología obsesivo compulsiva son el resultado de la activación del sistema inmune adaptativo por el estreptococo del grupo A. Se presenta el caso de un paciente masculino de 6 años, con cuadro de tres semanas de evolución de movimientos involuntarios en cabeza y miembros superiores, así como cambios de conducta, cuyo antecedente de importancia fue una infección respiratoria y en piel varias semanas previo al inicio de los síntomas

*Médico Pediatra en Hospital Tela

**Médico general de Emergencia Hospital Tela

*** Neurólogo Pediatra, IHSS-RN

Dirigir correspondencia a: ivan81hn@yahoo.com

Recibido: 20 de Diciembre 2017 Aprobado: 18 Julio 2018
PALABRAS CLAVES: Trastornos

Neuropsiquiátricos

Autoinmunes

Pediátricos Asociados a Estreptococo (PANDAS), Estreptococo beta Hemolítico del Grupo A (SBHGA), Trastorno Obsesivo Compulsivo (TOC), Corea de Sydenham ABSTRACT

PANDAS (Pediatric Autoimmune Neuropsychiatric Disorders Associated With Streptococcus) was created to describe a theoretical subgroup of cases of obsessive-compulsive disorders (OCD) and $/$ or tics in which the onset or exacerbation of symptoms is triggered by group A hemolytic streptococcus infection (GABHS). Although there is still no incidence data, it is estimated that the disorder affects approximately $1 \%$ of children or children under 12 years of age, because they are less likely to have antibodies against streptococci and are probably underdiagnosed. The existence of this disorder has generated considerable clinical and scientific advances, as well as controversy.

Pathophysiologically, PANDAS is proposed to be a poststreptococcal autoimmune disorder similar to Sydenham's chorea, hypothesizing more specifically that tics and obsessivecompulsive symptoms are the result of activation of the adaptive immune system by group A streptococcus. We present the case of a 6-year-old male patient, with a three-week history of involuntary movements in the head and upper limbs, as well as behavioral changes, whose history of importance was a respiratory and skin infection several weeks before the onset of the symptom. 
KEY WORDS: Pediatric Autoimmune Neuropsychiatric Disorders (PANDAS), Group A hemolytic streptococcus (SBHGA), obsessive-compulsive disorders (OCD), Sydenham's chorea.

\section{INTRODUCCIÓN}

Los PANDAS se originaron a partir del trabajo de observación de Swedo y colaboradores, quienes formalizaron su definición en 1998 en un conjunto de criterios operacionales. La aplicación de estos criterios, que se centra en tics y síntomas obsesivos compulsivos como síntomas centrales, ha encontrado dificultades, y finalmente conducen a una alta tasa de diagnósticos erróneos $^{(1-3)}$. La existencia de este trastorno ha generado considerables avances clínicos y científicos, así como controversia(4,5). Inicialmente, se cree que PANDAS podría tratarse de trastorno autoinmune postestreptocócico similar a la Corea de Sydenham y podrían ser dos presentaciones distintas de disfunción corticosubcortical desencadenada por SBHGA $(6,1)$. La Corea de Sydenham, una manifestación de fiebre reumática, se considera el prototipo de trastornos neurológicos causados por respuestas inmunes aberrantes a SBHGA. Estos pacientes a menudo exhiben TOC junto con otros anomalías del comportamiento. No existe aún total claridad acerca de los mecanismos implicados. Se cree que el contacto con el SBHGA en un paciente susceptible desencadena una respuesta inmune anormal que induce la formación de autoanticuerpos contra ganglios basales ${ }^{(7,8)}$.
Resulta de especial importancia que el manejo terapéutico se oriente con una visión integradora de equipo multidisciplinario, el cual siempre deberá contar con la presencia de un psiquiatra para procurar una terapéutica oportuna con psicofármacos si esta resultara necesaria ${ }^{(9)}$.

\section{CASO CLÍNICO}

Paciente masculino de 6 años de edad procedente de Aldea San Juan Pueblo, Atlántida; traído por sus padres con historia de presentar hace tres semanas, movimientos involuntarios en cabeza y ojos, así como cambios de comportamiento; volviéndose en ocasiones agresivo $y$ no obedece órdenes. Como antecedente de importancia, sus padres refieren que presentó lesiones en piel localizadas en tronco y miembros inferiores hace aproximadamente cinco semanas; tratado en centro de salud como impétigo con antibiótico cuyo nombre desconocen. A la exploración física: signos vitales dentro de rangos normales para su edad y sexo; Cooperador, inquieto, Glasgow 15, fuerza y sensibilidad conservada, con movimiento involuntarios en la cabeza de forma pendular,no nistagmos, marcha normal, romberg A.S.O a las tres semanas negativo. Manejándose como una encefalitis postinfecciosa y cita control en una semana. Paciente regresa al tercer día ya que presenta una exacerbación en cuanto a su sintomatología, con insomnio e intento de agresión física hacia la madre, 
por lo que es referido al neurólogo pediatra quien basado en las características clínicas y antecedentes patológicos del paciente decide manejarlo como un síndrome de PANDAS con amitriptilina $1 \mathrm{mg} / \mathrm{kg} /$ día durante tres a cuatro semanas, recomendaciones a los padres sobre identificación oportuna de signos y síntomas de alerta y / o pobre evolución clínica y evaluaciones semanales por médico pediatra; presentando una mejoría clínica significativa y aún en seguimiento mensual por consulta externa.

\section{DISCUSIÓN}

Este trastorno fue descrito aproximadamente hace más de 20 años por Kondo y Kabasawa en un paciente japonés de 11 años, que presentó súbitamente tics motores y vocales indistinguibles del Sd. de Tourette idiopático, después de una infección por estreptococo; sin respuesta al tratamiento convencional para los tics, pero con una gran mejoría con los corticoesteroides ${ }^{(1,2)}$. En 1998 Susan Swedo et al. Describieron las características clínicas de 50 niños TOC y/o tics en los que una infección por estreptococo beta hemolítico del grupo A desencadenó el inicio o la exacerbación de los síntomas. Por primera vez se creó el término de PANDAS. Posteriormente el NIMH (Instituto Nacional de Salud Mental de Estados Unidos) propuso los criterios diagnósticos ${ }^{(1,3,10,11)}$ (Ver Tabla 1) Inicialmente, se creyó que PANDAS podría tratarse de trastorno autoinmune postestreptocócico similar al de la Corea de Sydenham.
Tabla 1: CRITERIOS DIAGNÓSTICOS DE PANDAS SEGÚN LA NIMH

Criterios para PANDAS, segin el NIMH

1. Presencia de un trastorno obsesivo-compulsivo y/o tics (por criterios del DSM-IV).

2. Edad de comienzo entre los 3 años y la adolescencia.

3. Presentación abrupta de síntomas 0 evolución caracterizada por una acentuación dramática de los sintomas.

4. Exacerbación de síntomas temporalmente relacionada con la infección por estreptococo beta hemolitico del grupo A (SBHGA).

5. Examen neurológico anormal (hepiractividad, movimientos coreinformes y tics) durante la recaida

Fuente: DSM IV manual diagnóstico y estadistico de los trastornos mentales $4^{a} \mathrm{ed}$

Específicamente se hipotetizó que los tics y la sintomatología obsesivo compulsiva eran el resultado de la activación del sistema inmune adaptativo por el estreptococo hemolítico del grupo A (SBHGA), ya sea por la inducción de anticuerpos que reaccionan de forma cruzada contra el tejido neuronal (mimetismo molecular) o por la producción de proteínas secretadas que median y regulan la inmunidad y la inflamación (citoquinas y quimiocinas). Por lo tanto, para confirmar una etiología autoinmune en PANDAS, los investigadores intentaron identificar anormalidades en los marcadores de inmunidad(4,7).

Susan Swedo, propone que esta predisposición genética individual es evidenciable por la presencia del antígeno D8/17 identificado por métodos de detección de anticuerpos monoclonales, microscopía por inmunofluorescencia y citometría de flujo. Este antígeno es considerado marcador para PANDAS ya que está presente entre el 60,6 y $100 \%$ de los pacientes con esta entidad y no se modifica a lo largo del tiempo ni tampoco con infecciones repetidas por SBHGA. Es una inmunoglobulina de tipo $M$ que se encuentra formando parte de los linfocitos $B$ y determina una respuesta de reacción cruzada por mimetismo molecular (los epítopes de la proteína M del SBHGA son similares a algunas partes del citoesqueleto, núcleo y membrana de las neuronas de los 
ganglios basales). Se crean así autoanticuerpos $\lg G$ denominados anticuerpos antineuronales que producirían una reacción inflamatoria tipo II que desencadenaría finalmente los síntomas clínicos (1,10). También se ha postulado la posible existencia de PANDAS en la edad adulta, tanto como primera manifestación o como expresión continuada de la patología pediátrica. La explicación de este fenómeno se basa en:

- Títulos altos de ASLOs, anticuerpos antiganglios basales y antígeno D8/17 en población adulta con el trastorno de La Tourette y trastorno obsesivo compulsivo descrito por Church y otros autores.

- La presencia de anticuerpos que proveen protección contra infección estreptocócica en el $98 \%$ de los sujetos mayores de 12 años en los estudios de Fishetti(2). Clínicamente en los pacientes pediátricos generalmente los síntomas se presentan de forma abrupta y "dramática", generalmente en las noches; es más frecuente en el sexo femenino, con una edad de inicio de los síntomas entre los 3 y 12 años de edad; todas estas manifestaciones clínicas frecuentemente se encuentran precedidas por una amigdalitis estreptocócica, este último elemento resulta de gran importancia y debe ser precisado en el interrogatorio inicial

Estudios recientes sugieren un riesgo asociado con infecciones repetidas por Estreptococo del grupo A (SBHGA) en niños que tienen síntomas neuropsiquiátricos. Por ejemplo, un historial de múltiples infecciones por SBHGA dentro de un período de un mes se asoció con un mayor riesgo de síndrome de Tourette $(\mathrm{OR}=13.6 ; 36)$. Otra fuente descubrió que la cantidad de infecciones anteriores por SBHGA se relaciona positivamente con la gravedad, curso e incidencia de la recaída $(7,11)$

\begin{tabular}{|l|l|}
\hline Cultivo faringeo: & $\begin{array}{l}\text { Prueba estándar o de referencia para el } \\
\text { diagnóstico de infección por SBHGA. El resultado } \\
\text { se obtiene a las 24-48 horas. En condiciones } \\
\text { ideales la sensibilidad del cultivo es del 90-95\% y } \\
\text { la especificidad llega a ser del } 99 \% .\end{array}$ \\
\hline $\begin{array}{l}\text { Cuantificación de los niveles de antiestreptolisina } \\
\text { (ASL }\end{array}$ & $\begin{array}{l}\text { Métodos de detección rápida (3-10 minutos). Se } \\
\text { utilizan los títulos seriados de ASLO si el cuadro } \\
\text { infeccioso lleva más de una semana. }\end{array}$ \\
\hline Cuantificación de anticuerpos anti DNASA B: & $\begin{array}{l}\text { Es la prueba más especifica y sus niveles duran } \\
\text { más tiempos elevados, pero la más costosa y poco } \\
\text { útil como prueba de tamizaje. }\end{array}$ \\
\hline
\end{tabular}

\section{TRATAMIENTO - Antibióticos}

El uso de antibióticos solo está indicado en el manejo de la infección aguda por SBHGA. Sin embargo la evidencia no permite concluir que los antibióticos mejoren los síntomas neuropsiquiátricos una vez establecidos, por lo que no se recomienda su uso de forma profiláctica ${ }^{(6)}$. La pauta recomendada para la faringoamigdalitis aguda según la Asociación Española de Pediatría de Atención Primaria 2011 es: -Penicilina V oral de 25-50 mg/kg/ día, cada 8-12 horas por 10 días. En caso de vómitos 0 intolerancia oral, falta de cumplimiento, se recomienda Penicilina G Benzatinica IM en dosis única: • $600.000 \mathrm{U}$ en los menores de 12 años y < $27 \mathrm{~kg}$. • 1.200.000 U en los mayores de 12 años o $\geq 27 \mathrm{~kg}$. -Amoxicilina: actualmente muy utilizada por la mayor variedad de presentaciones farmacéuticas, su mejor sabor y tolerancia. Es equivalente en cuanto a eficacia clínica y bacteriológica e incluso superior en la prevención de recaídas a penicilina.Dosis: $50 \mathrm{mg} / \mathrm{kg} /$ día cada 8012 horas por 10 días. 


\section{Terapia inmunomoduladora}

Actúan desviando la respuesta inmune o bloqueando la respuesta mediada por anticuerpos (2-4).

- Inmunoglobulina intravenosa: mezcla de IgG proveniente de diversos donantes.

- Plasmaféresis: separa los elementos formes y solubles de la sangre del paciente, reemplazando la fracción soluble por una solución isotónica carente de anticuerpos, citoquinas, compuestos de complemento, hormonas y proteínas séricas (1). Los resultados preliminares de un estudio realizado por Perlmutter mostraron que estos tratamientos pueden ser efectivos para acelerar la recuperación de los pacientes, lo cual ayudaría a confirmar el origen autoinmune del PANDAS (2).

\section{Terapias psicológicas y psicofármacos (2, $5,9)$}

Se utilizan para el manejo de síntomas neuropsiquiátricos según cada paciente. Por ejemplo, en el caso de síntomas obsesivo-compulsivos se combina la terapia cognoscitiva conductual con fármacos inhibidores de la receptación de serotonina.

\section{REERENCIAS}

BIBLIOGRÁFICAS

1.Bacic $M$, Carcamo P, PANDAS: Enfermedad Pediátrica Neuropsiquiátrica Autoinmune Asociada a Infección por Estreptococo (Rev GPU 2015; 11; 4: 379-382).

2.Gabbay V, Coffey B, Babb J; et al , Pediatric Autoimmune Neuropsychiatric Disorders Associated With Streptococcus: Comparison of Diagnosis and Treatment in the Community and at a Specialty Clinic, Pediatrics 2008;122: 273-278.
3.Pupillo J, PANDAS/PANS treatments, awareness evolve, but some experts skeptical, AAP News March 28, 2017, https://www.pandasppn.org/ diagnosticguidelines/

4. Singer $\mathrm{H}$, Gause $\mathrm{C}$, Morris $\mathrm{C}$, et al, Serial Immune Markers Do Not Correlate With Clinical Exacerbations in Pediatric Autoimmune Neuropsychiatric Disorders Associated With Streptococcal Infections, Pediatrics 2008;121:1198-1205.

5.Chávez O, Crespo K, De Acha R, Flores A. Desorden neuropsiquiátrico pediátrico asociado con infecciones estreptocócicas. Rev Cient Cienc Med 2010; 13(2): 86-89. 6.Macerollo A, Martino D. Pediatric autoimmune neuropsychiatric disorders associated with streptococcal infections (PANDAS): an evolving concept. Tremor Other Hyperkinet Mov 2013; 3: http:// tremorjournal.org/article/view/167.

7.Delucchi V, Pavlidis E, Piccolo B, Pisano, Febrile and PostinfectiousMyoclonus: Case Reports and Review of the Literature, Neuropediatrics 2015; 46:26-32.

8.Scolnick B, Pediatric Autoimmune Neuropsychiatric Disorders Associated With Streptococcus, PEDIATRICS Volume 123, Number 1, January 2009.

9.Tona J, Posner T, Pediatric Autoimmune Neuropsychiatric Disorders: A New Frontier for Occupational Therapy Intervention, NOVEMBER 14, 2011 -WWW.AOTA.ORG. 10.Swedo SE, Leckman JF, Rose NR, and From Research Subgroup to Clinical Syndrome: Modifying the PANDAS Criteria to Describe PANS (Pediatric Acute-onset Neuropsychiatric Syndrome). Pediatr Therapeut 2:113. doi:10.4172/2161-0665.1000113 11. Murphy $\mathrm{T}$, Storch $\mathrm{E}$, Lewin $\mathrm{A}$, et al, Clinical Factors Associated with PANDAS, J Pediatr. 2012 February; 160(2): 314-319. doi:10.1016/j.jpeds.2011.07.012. 